\title{
Women's Perceptions and Practices on Human Papillomavirus Infection and Human Papillomavirus Vaccine in Sri Lankan Community
}

${ }_{1}^{1}$ Chinthika P Gunasekara, ${ }^{2}$ Irangi Karunanayake, ${ }^{3}$ Nirasha Premathilake, ${ }^{4}$ Nawashika H Senarathna,

${ }^{5}$ Ishanka Udayangani, ${ }^{6}$ Manjula M Weerasekara, ${ }^{7}$ Ramya Pathiraja, ${ }^{8}$ Neluka Fernando

\begin{abstract}
Aim: This study investigated the knowledge, perceptions, and practices regarding human papilloma virus (HPV) infection among women attending gynecology clinic and antenatal clinic at a teaching hospital in Sri Lanka.
\end{abstract}

Materials and methods: A cross-sectional survey was conducted of 400 women attending gynecology and antenatal clinics at a teaching hospital between September 2015 and December 2015. A pretested self-administered questionnaire was used to collect data. A score of $\geq 75 \%$ was considered as good while $74-50 \%$ was considered as moderate and $<50 \%$ as poor.

Results: Out of 400 women who participated in this study, the percentage of respondents who had heard of HPV, cervical cancer (CC) and genital warts were $23 \%, 80 \%$, and $41 \%$ respectively. However, only $16 \%$ knew that there is an association between HPV and CC. Although $48 \%$ believed that CC is common in Sri Lanka, only $6.8 \%$ considered themselves at risk. Overall knowledge of risk factors, symptoms, transmission and diagnosis was 44\% (CC) and 14\% (HPV infection) respectively. Only $14.3 \%$ was aware of the availability of the HPV vaccine in Sri Lanka. None of the participants in the study group had received the HPV vaccine.

Conclusion: This population had poor knowledge, attitudes, and practices with regard to HPV infection.

Clinical significance: Therefore substantial effort should be made to educate the society regarding HPV infection, complications, and preventive measures.

\footnotetext{
${ }^{1,6,7}$ Senior Lecturer, ${ }^{2,3}$ Research Student, ${ }^{4,5}$ Demonstrator, ${ }^{8}$ Professor

1,4-6,8 Department of Microbiology, Faculty of Medical Sciences, University of Sri Jayewardenepura, Nugegoda, Democratic Socialist Republic of Sri Lanka

${ }^{2,3}$ Department of Allied Health Sciences, Faculty of Medical Sciences, University of Sri Jayewardenepura, Nugegoda, Democratic Socialist Republic of Sri Lanka

${ }^{7}$ Department of Obstetrics and Gynaecology, Faculty of Medical Sciences, University of Sri Jayewardenepura, Nugegoda, Democratic Socialist Republic of Sri Lanka
}

Corresponding Author: Chinthika P Gunasekara, Senior Lecturer, Department of Microbiology, Faculty of Medical Sciences, University of Sri Jayewardenepura, Nugegoda, Democratic Socialist Republic of Sri Lanka, e-mail: chinthika@ sjp.ac.lk
Keywords: Attitudes, Cervical cancer, HPV vaccine, Human papillomavirus, Knowledge, Practices, Sri Lanka.

How to cite this article: Gunasekara CP, Karunanayake I, Premathilake N, Senarathna NH, Udayangani I, Weerasekara MM, Pathiraja R, Fernando N. Women's Perceptions and Practices on Human Papillomavirus Infection and Human Papillomavirus Vaccine in Sri Lankan Community. J South Asian Feder Obst Gynae 2019;11(1):65-69.

\section{Source of support: Nil}

Conflict of interest: None

Date of received: $02-08-2016$

Date of acceptance: $12-10-2018$

Date of publication: March 2019

\section{INTRODUCTION}

Cervical cancer is a global health burden being the fourth most common cancer in women in the world. ${ }^{1}$ It is the second commonest cancer in the less developed regions with a reported very high motility $(87 \% 2){ }^{2}$ The HPV infection is the commonest cause for cervical cancers). ${ }^{3}$ However, unlike most cancers, cervical cancer due to HPV can be prevented effectively by vaccination.

An HPV is a sexually transmitted virus infecting $50-80 \%$ of sexually active women during their lifetime. ${ }^{4}$ Most infections are subclinical and transient, but persistent infection by oncogenic HPV subtypes increase the risk of developing genital warts and cervical cancer. HPV subtypes 16 and 18 are responsible for $70 \%$ of cervical cancers reported. ${ }^{5}$

Prophylactic vaccination against HPV is highly effective in preventing cervical cancers. Currently, there are two HPV vaccines; Quadrivalent (against HPV subtypes 6,11,16 and 18) and bivalent (against HPV subtypes 16 and 18). ${ }^{6}$ The quadrivalent vaccine is protective against both cervical cancer and genital warts, whereas bivalent vaccine is only protective against cervical cancer.

The HPV vaccine was introduced in Sri Lanka in 2006 but is not included in the extended program of immunization (EPI). ${ }^{7}$ Because it is a non-EPI vaccine, it's public access is limited by economy, awareness, and attitudes of the local population. These facts are important in influencing the adults of Sri Lanka who are the decision-makers 
in immunizing their children as the target population of vaccination is young adolescents.

It is unfortunate that while effective prophylactic measures are available, cervical cancers are still a leading cause of death among women globally. Effective use of prophylactic measures is hampered by a lack of adequate knowledge of HPV and cervical cancer among women of child-bearing age. The inclusion of HPV vaccine into the EPI schedule in Sri Lanka is expected in the near future. However, the success of that program will depend on the acceptance of vaccination by the population who will be influenced by their knowledge and attitudes. Therefore, it is vital to assess the perceptions and practices of the local population to cater to effective vaccination and cervical cancer screening programs.

\section{MATERIALS AND METHODS}

A descriptive cross-sectional study was carried out on 400 patients selected from gynecology and antenatal clinics of Colombo South Teaching Hospital who were more than 18 years, not mentally retarded and not illiterate. Systematic sampling was done in which every second patient entering the clinic was included in the study. After obtaining informed consent self-administered questionnaire was used as a study instrument to obtain the details which were pretested on 25 random patients. The questionnaire was composed of 4 parts. Demographic data were obtained from part through 18 questions. Knowledge with regard to cervical cancer, HPV infection and HPV vaccine was assessed via 34 questions in part $B$. In part C, 12 questions were included to determine attitudes regarding HPV vaccine and part $\mathrm{D}$ was to ascertain practices regarding HPV vaccine using 13 questions.

Statistical Package for Social Science (SPSS) was used to analyze the data and to assess the significance of the statistical relationship between variables according to the $p$ value. Assessment of knowledge, attitudes, and practices was done by using a scoring system in which one point was given for correct knowledge, good attitudes and good practices, while 0 points for incorrect knowledge, poor attitudes, and poor practices. Percentage of each category was calculated using the average score of each category. Seventy-five percent for knowledge, attitudes, and practices was considered as good, $50-74 \%$ as moderate while less than $50 \%$ was considered as poor. ${ }^{8,9}$

\section{Ethical and Humane Consideration}

An ethical review committee of the University of Sri Jayewardenepura (B Pharm 4/2015) and the Colombo
South Teaching Hospital (no. 451) granted the ethical approval for the study.

\section{RESULTS}

\section{Demographic Data}

Out of the 400 participants, 176 (44\%) were pregnant women, and 224 (56\%) were nonpregnant women. The majority (90\%) were of Sinhalese ethnicity while 5\%, 4.3\%, and $0.8 \%$ were Tamils, Muslims and other ethnic groups, respectively. Fifty-seven percent of the study group were educated up to general certificate of education (GCE) advanced level and only $9.3 \%$ had received an education below GCE. Ordinary Level. Mean age of participants were 37.33 years. The number of participants who have children was 256 (64\%) of them, 190 (47.5\%) were females.

\section{Knowledge}

Out of the participants $80 \%$ had heard of cervical cancer while $41 \%$ had heard of genital warts. Even though $48 \%$ knew that cervical cancer is common in Sri Lanka, the majority (93.3\%) did not consider themselves at risk. A statistically significant number of participants $(p>0.05)$, who had heard of cervical cancer had secondary or tertiary education. Participants, who had a tertiary education, had obtained the highest score for having heard of cervical cancer $(95.5 \%)$. Of the participants, $60.3 \%$ were aware that cervical cancer was fatal, but its preventability was only known by $36.8 \%$. Even though $13.8 \%$ of the participants were aware that cervical cancer could be detected early using a screening test, only $33.5 \%$ of them knew that the screening test was the Pap smear.

Of the study population, $23 \%$ had heard of HPV, yet only $5.5 \%$ thought that they could get infected. While $26.8 \%$ answered that HPV is a sexually transmitted disease, $26.5 \%$ stated that it is life-threatening. Those with only primary education had never heard of HPV $(100 \%)$ and the majority of those with secondary (84.3\%) and tertiary (58.2\%) education also had not heard about HPV. Among the participants, 42\% knew early-stage cervical cancer symptoms such as unusual vaginal discharge, spotting, and lower abdominal pain. Only 16\% were aware of the association between HPV infection and cervical cancer.

Most of the participants $(86.3 \%)$ did not know that HPV infection could be prevented. A statistically significant difference $(p>0.05)$ was observed among participants who were aware $(36.3 \%)$ of the cervical cancer screening test and those who were unaware $(63.8 \%)$ of it. Those with tertiary education had a better knowledge (56\%) in this regard in comparison with those who had primary $(13.5 \%)$ or secondary education (28.4\%). 


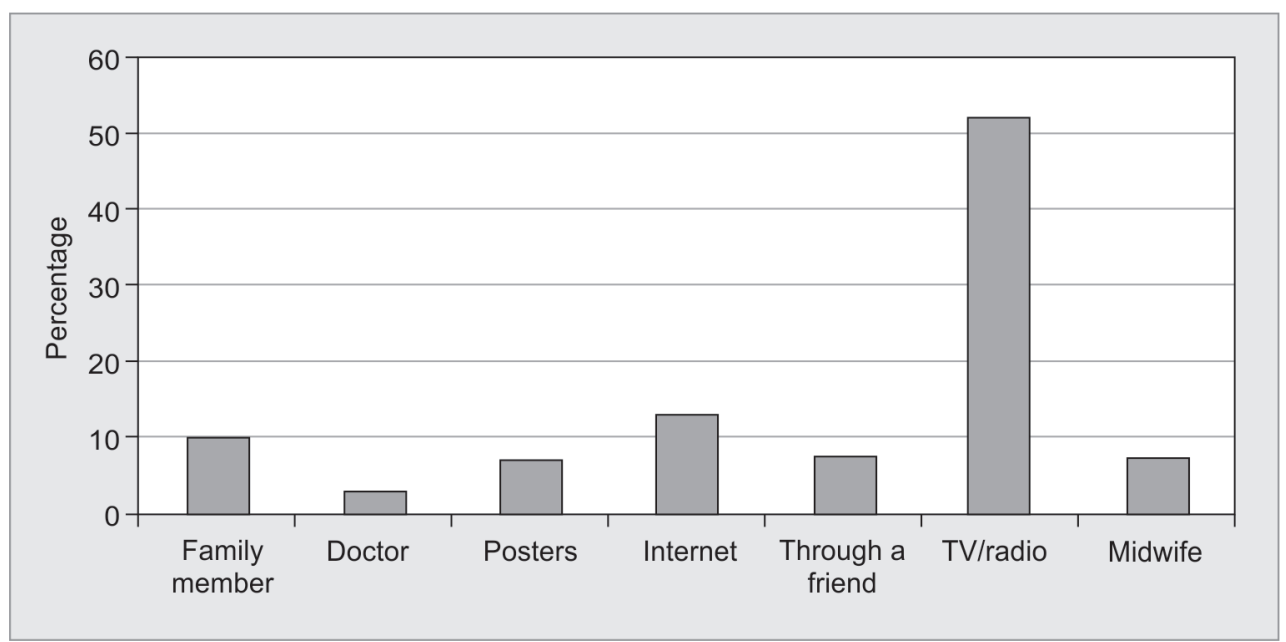

Graph 1: Participants' source of information on HPV infection and HPV vaccine

Only $14.3 \%$ knew that there is a vaccine available to prevent cervical cancer. Participants had received information on HPV infection and vaccine from several sources (Graph 1). Most frequent sources of knowledge were TV and radio programs $(10.6 \%)$ while healthcare professionals were a considerably poor source of information (Graph 1).

The overall knowledge of cervical cancer when determined was poor among $57.68 \%$, while $20.56 \%$ were 'moderate' and $21.56 \%$ were 'good'. According to the total scores, the level of knowledge on HPV infection of the participants was good $(7.3 \%)$, moderate $(9.2 \%)$ and poor $(83.5 \%)$. The overall knowledge on HPV vaccine was good $(9.3 \%)$, moderate $(4.3 \%)$ and poor $(86.5 \%)$.

\section{Attitudes}

When considering the overall responses of attitudes, the majority $(69.66 \%)$ of the participants had 'poor' attitudes, $19.67 \%$ had a 'moderate' and $10.67 \%$ had 'good' attitudes. Only a few participants believed that HPV vaccination was the best method to prevent HPV infection (19\%). While the cost of the vaccine was a concern, $51.3 \%$ stated they would get the vaccine if it was freely available.
Alarmingly only $34.5 \%$ of participants desired to get their children vaccinated. Most (80.3\%) believed that the availability of the information regarding the HPV vaccine was inadequate. Only $47.8 \%$ believed that it is better to get a Pap smear test done after 40 years of age by a woman annually. Meanwhile, $31.3 \%$ was not interested in getting a Pap test done as they believed they are not at risk. Of the participants, $31.5 \%$ believed that the HPV vaccine would help to protect them from cervical cancer (Table 1).

\section{Practices}

In the study group, only 50 participants $(12.5 \%)$ had ever done a Pap test out of which 33 participants $(66 \%)$ had undergone the pap smear on the recommendation by medical personnel. Only 17 participants (34\%) had the pap smear done due to their desire. None $(0 \%)$ had received the HPV vaccine, and the majority (69.3\%) did not know of the existence of it.

\section{DISCUSSION}

Knowledge, attitudes, and practices of HPV infection and HPV vaccine among reproductive-aged women

Table 1: Responses of patients to statements used to assess attitude

\begin{tabular}{ll}
\hline Attitude assessed & Positive attitude (\%) \\
\hline HPV vaccination is the best method to prevent HPV infection & 19 \\
I am willing to be vaccinated if the vaccine is freely available & 51.3 \\
I think it is important for my child to get vaccinated & 34.5 \\
I think that the availability of the information regarding the HPV vaccine is not adequate & 80.3 \\
I would much prefer to have other preventive methods rather than getting the HPV vaccine & 14.5 \\
I think I should get the pap smear test done after 40 years of age once a year & 47.8 \\
I do not think I am likely to get cervical cancer so I am not worried & 31.3 \\
I should ask a doctor whether I am at risk of cervical cancer & 48.3 \\
I am not at risk of getting cervical cancer & 20.3 \\
I believe that HPV vaccine will help to protect me from cervical cancer & 31.5 \\
\hline
\end{tabular}


in Sri Lanka were assessed in this study. The results of our study indicate a lack in knowledge regarding the consequences of HPV infection, its association with cervical cancer, availability of screening tests for early detection of cervical cancer and prophylactic vaccination and availability.

Currently, two types of HPV prophylactic vaccines are available as quadrivalent and bivalent vaccines in the private sector in the country. However, the cost of the vaccine is an important barrier to vaccination. Further the vaccine is still being considered for inclusion in the national immunization programme and is therefore not freely available to the public. Increased awareness of the vaccine among the population will result in increased acceptance of the vaccine by the community.

The average level of knowledge, attitudes, and practices on cervical cancer, HPV infection and HPV vaccine were poor among women attending to gynecology clinic and antenatal clinics at Colombo South Teaching Hospital which was far below our expectations. In this study population, more than $50 \%$ of women had completed their GCE ordinary level examination. However, their knowledge related to this important health problem was poor. Hence this study is important in alerting the relevant health authorities in the country. The role of doctors and midwives in promoting awareness also needs to be strengthened.

The study of Johson et al. conducted among women in Nepal showed that only $53.3 \%$ of women were aware of cervical cancer, and $15.4 \%$ had heard of $\mathrm{HPV}^{10}$ which is similar to our findings. However, studies done in East Asian countries report a better knowledge ${ }^{11-13}$ which suggest that these countries may be having better health awareness programs. Improved awareness about cervical cancer, strongly contributes to the use of the HPV vaccine as a preventive tool.

Awareness of HPV as a sexually transmitted disease was poor in our population $(26.8 \%)$, yet a study done among Sri Lankan university students in 2015 showed $47 \%$ awareness. ${ }^{14}$ Indeed as seen by these results the awareness of HPV and cervical cancer were highest among those having tertiary education. Similarly, awareness of HPV as a sexually transmitted disease was found to be better in developed countries such as in Germany. ${ }^{15}$

Prophylactic vaccination against HPV is currently the best preventive method for cervical cancer. ${ }^{16}$ The study of Markowitz et al. in the United States showed that within 6 years of vaccine introduction, there was a $64 \%$ decrease in HPV prevalence among females ${ }^{17}$ emphasizing that vaccination could reduce the prevalence of HPV infection. Bivalent HPV vaccine efficacy was $90 \%$ in preventing HPV type 16 or type $18 .{ }^{18}$ However, it is important to note that very few participants knew about the availability of HPV vaccine in Sri Lanka. Unfortunately, no one in this study had been vaccinated with the HPV vaccine. The lack of perception of the severity of the outcomes of HPV infection could be one reason behind the negligence seen. Further, the cost incurred and the unavailability of the vaccine in the free national immunization program in the country are considered as major barriers to vaccination.

\section{CONCLUSION}

In conclusion, the study indicated a poor knowledge, attitudes, and practices among participants regarding cervical cancer, HPV infection and HPV vaccine. Therefore substantial effort should be made to educate the society regarding HPV infection, complications, and preventive measures.

\section{ACKNOWLEDGMENT}

The authors would like to thank the staff of the Antenatal and gynecology clinics at Colombo South Teaching Hospital.

\section{REFERENCES}

1. Organization WH. Cervical Cancer Estimated Incidence, Mortality and Prevalence Worldwide in 2012. GLOBOCAN 2012 (IARC), Section of Cancer Surveillance; 2012.

2. Ferlay J, Soerjomataram I, Dikshit R, Eser S, Mathers C, Rebelo M, et al. Cancer incidence and mortality worldwide: Sources, methods and major patterns in GLOBOCAN 2012. International Journal of Cancer. 2015;136(5):E359-E386.

3. Forman D, de Martel C, Lacey CJ, Soerjomataram I, LortetTieulent J, Bruni L, et al. Global burden of human papillomavirus and related diseases. Vaccine. 2012 Nov 20;30:F12-F23.

4. Baseman JG, Koutsky LA. The epidemiology of human papillomavirus infections. Journal of Clinical Virology. $2005 \mathrm{Mar}$ 1;32:16-24.

5. Smith JS, Lindsay L, Hoots B, Keys J, Franceschi S, Winer R, et al. Human papillomavirus type distribution in invasive cervical cancer and high-grade cervical lesions: a meta-analysis update. International Journal of Cancer. 2007 Aug 1;121(3):621-632.

6. Karunaratne K. Prophylactic HPV vaccination-current status. Sri Lanka Journal of Obstetrics and Gynaecology. 2012;32(2):25-26.

7. Ministry of Health, Government of the Democratic Socialist Republic of Sri Lanka. Comprehensive Multi-year Plan for Immunization 2012-2016.

8. Ariyaratne MHJD Gunasekara TDCP, Weerasekara MM, Kottahachch J, Kudavidanage BP, Fernando SSN. Knowledge, attitudes and practices of hand hygiene among final year medical and nursing students at the University of Sri Jayewardenepura. Sri Lankan Journal of Infectious Diseases. 2013;3(1):15-25.

9. Suchitra JB, Lakshmi Devi N. Impact of education on knowledge, attitudes and practices among various categories of health care workers on nosocomial infections. Indian Journal of Medical Microbiology. 2007 Jul;25(3):181-187. 
10. Johnson DC, Bhatta MP, Gurung S, Aryal S, Lhaki P, Shrestha S. Knowledge and awareness of human papillomavirus (HPV), cervical cancer and HPV vaccine among women in two distinct Nepali communities. Asian Pacific Journal of Cancer Prevention: APJCP. 2014;15(19):8287-8293.

11. Wang SM, Zhang SK, Pan XF, Ren ZF, Yang CX, Wang ZZ, et al. Human papillomavirus vaccine awareness, acceptability, and decision-making factors among Chinese college students. Asian Pacific Journal of Cancer Prevention: APJCP. 2014;15(7):3239-3245.

12. Ezat SW, Hod R, Mustafa J, Mohd D, Ahmad ZH, Sulaiman AS, et al. National HPV immunisation programme: knowledge and acceptance of mothers attending an obstetrics clinic at a teaching hospital, Kuala Lumpur. Asian Pacific Journal of Cancer Prevention. 2013;14(5):2991-2999.

13. Abudukadeer A, Ding Y, Niyazi M, Ababaikeli A, Abudula A. Distribution of HPV genotypes in uterine cervical lesions among the Uighur women in Xinjiang province of China. European Journal of Gynaecological Oncology. 2010 Jan 1;31(3):315.

14. Östh J. Knowledge of Human Papilloma Virus, Cervical Cancer and Cytological Screening and Attitudes towards and Practices of Screening among Undergraduate students at Rajarata University, Sri Lanka: A cross-sectional study. 2015.

15. Stöcker P, Dehnert M, Schuster M, Wichmann O, Deleré Y. Human papillomavirus vaccine uptake, knowledge and attitude among 10th grade students in Berlin, Germany, 2010. Human Vaccines and Immunotherapeutics. 2013;9(1):74-82.

16. Kaarthigeyan K. Cervical cancer in India and HPV vaccination. Indian Journal of Medical and Paediatric Oncology: Official Journal of Indian Society of Medical \& Paediatric Oncology. 2012 Jan-Mar;33(1):7-12.

17. Markowitz LE, Liu G, Hariri S, Steinau M, Dunne EF, Unger ER. Prevalence of HPV After Introduction of the Vaccination Program in the United States. Pediatrics. 2016 Mar;137(3):e20151968.

18. Paavonen J, Jenkins D, Bosch FX, Naud P, Salmeron J, Wheeler $\mathrm{CM}$, et al. Efficacy of a prophylactic adjuvanted bivalent L1 virus-like-particle vaccine against infection with human papillomavirus types 16 and 18 in young women: an interim analysis of a phase III double-blind, randomised controlled trial. Lancet (London, England). 2007 Jun 30;369(9580):2161-2170. 